\title{
THE WAGES SYSTEM IN ROMANIA - BETWEEN CONVERGENCE AND DISCRIMINATION. SUSTAINABILITY OR OVER-REGULATION?
}

\author{
Suzana Demyen ${ }^{1}$, Mirela Minică², Carmen Năstase ${ }^{3}$ \\ 1,2 Department of Business Administration - Reșița, Faculty of Economics and Business \\ Administration, Babeș - Bolyai University, Romania \\ 3 Faculty of Economics and Public Administration,"Ștefan cel Mare" of Suceava, Romania \\ suzana.demyen@ubbcluj.ro \\ mirela.minica@ubbcluj.ro \\ carmenn@seap.usv.ro
}

\begin{abstract}
The wages system in Romania is a subject of great importance. Although, apparently, the situation on the labour market has improved during the last years, compared to the member countries of the European Union there are many problems in Romania, due to an inadequate structure in the national economy, the way in which privatization and economy restructuring were performed, the mass emigration labour market, which have generated imbalances between labour supply and demand. The paper aims at highlighting the changes in the salary system in Romania in the period of post-accession to the European Union. An analysis was conducted, the official statistical data on the evolution of the average net monthly salary were detailed, differentiated by size classes of economic agents, by gender and by sectors of the national economy.
\end{abstract}

Keywords: labour market, discrimination, wages, public sector, private sector

JEL Classification: E24, E64, J31, J45, J71

\section{Introduction}

The transition to the market economy of the former socialist economies and the European economic integration have triggered important transformations in the labour market, which have accentuated or diminished its discrimination and vulnerabilities. The issue of the sustainability of an economic system automatically implies the trend towards balance of the labour market from the perspective of demand, supply and salary level.

According to literature, "macroeconomic stability is characterized by improving business conditions" (Belas et al., 2020). On ther other hand, the labour market can be seen as a system that has as input the demand and supply of labour and as outputs the income generated for employees and implicitly the expenses borne by employers. The feedback relationship between these elements can generate the system sustainability or, on the contrary, major imbalances on the labour market, such as unemployment (Pauhofova and Stehlikova, 2018) and underemployment.

The labour market reveals the processes and laws that ensure the demand and supply of labour, the mechanisms and operations underlying employment and efficient use thereof (Minică, 2004).

According to the neoclassical model, Dornbusch and Fisher (1990) highlight the conditions for achieving equilibrium in the labour market from the perspective of a microeconomic analysis. Companies will not require hours of work if there is no market demand for the goods and services offered. The labour demand is determined by the level of marginal labour productivity which is a decreasing quantity. All active and fit people make a choice between work and rest (non-work). The decision to work depends on two effects: income and substitution. 


\section{Conceptual framework}

As per the analysis conducted by Santos and Sequeira (2013), the level of the average wages (Marek, 2019) differs between the branches of activity, in industry there is an average hourly cost between 27.4 and 33.2 euros, while in construction it varies between 25 and 27.6 euros, and in services between 27 and 29.6 euros. The share of social contributions of employees in the total cost of labour is $23.7 \%$ on the average in the European Union. The pay gap between women and men is also due to the fact that $31.7 \%$ of employed women work part-time, while the European average of men employed in this system reaches only $8.8 \%$.

Vacas-Soriano, Fernández-Marcias and Muňos de Bustillo (2019) identify the fact that "mainstream theories" (also mentioned by Privarova, 2007) of economic growth (Ucak, 2012) predict a process of convergence as a result of the European economic integration. According to the theory of Heckscher-Ohlin, countries will specialize in those activities that efficiently capitalize on their resources, and the Stopler-Samuelson theory predicts that there will be a simultaneous process of convergence between countries in the price of production factors (labour and capital). The literature provides empirical evidence highlighting convergence between different groups of countries, known as convergence clubs (Baumol and Wolf, 1988), for countries with similar social institutions, economic reality and geographical positioning, and in particular for the European Union (Sachs and Warner, 1996). According to Radlinska et al, "global crises result in difficulties on the European labor markets" (Radlinska et. al, 2020).

The evolution of revenues is pro-cyclical and in conditions of recession their convergence process is distorted, especially due to the strong impact it had on countries with a low level of economic development (Chirinko, 1980; Brandolini, 2007).

Over time, due to the so-called model of social convergence, the differences between countries in terms of income have decreased (Clark, 2013) for the following reasons: modernization and standardization of institutions as a result of economic development (Meyer et al. 1997), the limits imposed by the structural variations of society by establishing a common division of labour (Levy, 1966); the influence of globalization in terms of technology (Bhalla, 2002), common policies and benchmarking applied to the European Union (Torfason and Ingram, 2010).

The gender of the employee has a negative effect in all countries, the consequence being that, on the level of the European Union, in 2017, women earned $16.2 \%$ less than men in terms of gross hourly income. The most important differences are registered in Estonia (25.3\%), the Czech Republic (28.1\%), Germany (21.5\%). On the other hand, the smallest differences between the gains obtained by men and women were registered in Romania $(5.2 \%)$, Italy (5.3\%), Luxembourg (5.5\%) and Belgium (6.1\%). Literature addresses this issue in terms of gender discrimination (Hedija and Musil, 2020; Gori et al, 2018; Macarie and Moldovan, 2012). Some of these income disparities can be explained by the individual characteristics of male and female employees (experience and education) and part of the gender segregation of the occupational sector (Rakauskiene and Chlivickas, 2007), the income gap (Zeman, 2019; Șandor et al, 2011) having a connection with cultural, legislative, social and economic factors, behind pay differences for the same work.

In 2018 , on the level of the EU member states, the average hourly labour cost ranged from 5.4 euros to 43.5 euros, the lowest levels being recorded in Bulgaria and Romania, and the highest in Denmark, Luxembourg and Belgium, according to the following graph: 


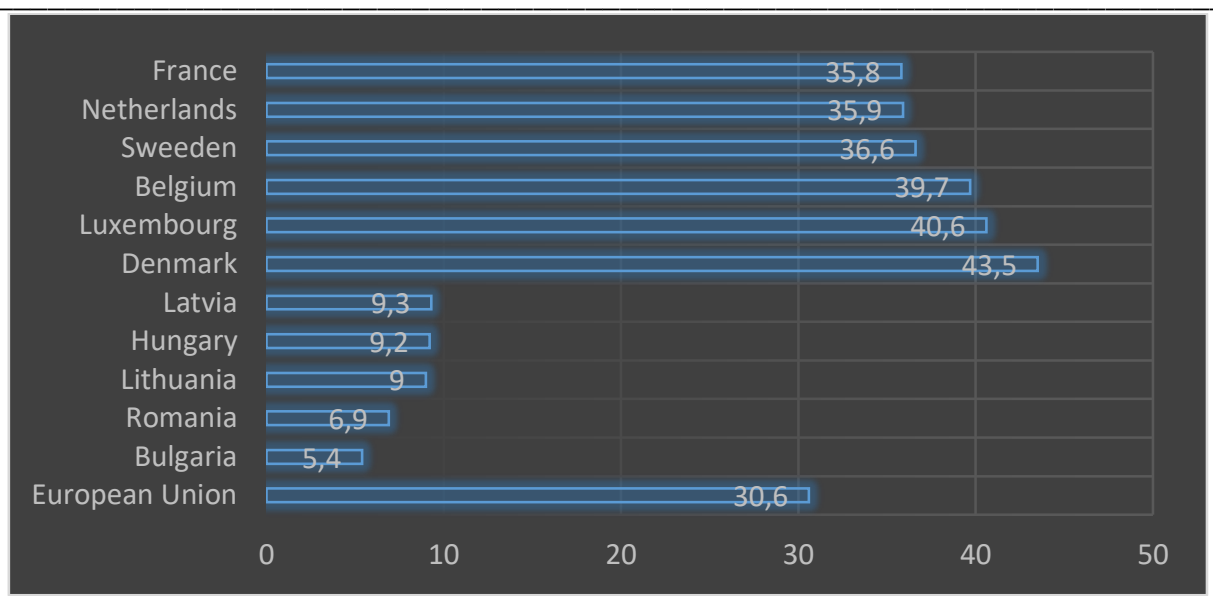

Figure 1: Average wage cost in several EU countries (euro)

Source: elaborated by the authors, according to Eurostat information

Budria and Moro-Egido (2014) state that the phenomenon of overqualification may reflect a "significant mismatch between the real potential of employees and the productivity limit at work". Hitka et al. (2018) mention flexibility related to family as a reason of discrimination of women ar workplace, while Vargic and Luptakova (2003) consider "building and supporting the existence of informal relationships" as being a positive aspect.

Due to the impact of the free movement of labour, on the European market and including in Romania, some vulnerabilities are created for certain categories of workers, especially for those with a low level of education (Gottvald et al, 2013). The importance of education is highlighted in literature (Vaiciukeviciute et al, 2019). According to Eurostat data, the number of workers with higher education in the EU Member States of the European Union in the period 2008-2015 increased by 13 million workers, while the number of those with secondary education decreased by 7.4 million. The high share of people with lower education is a major vulnerability of the labour market, especially in the economic crisis, and lifelong learning, which could reduce the effects of this situation, is formal, with no real impact on the level of training. Although many companies offer various courses and trainings in order to develop skills specific to the field of activity, they are financed from the companies' own resources, being an extra-salary cost of labour, which can put pressure on the salary fund and condition the employee to stay a certain period at the respective workplace, in the opposite situation, having to pay the equivalent value of these trainings. The most worrying phenomenon on the labour market in Romania, however, remains the decrease in labour supply, due to the massive international migration in recent years. Thus, according to the data of the National Institute of Statistics, 2.069 million people changed their usual residence, between 2008 and 2017, amid a decrease in the resident population, from 20.64 million in 2008 to 19.52 million in 2018.

\section{Empirical analysis}

\subsection{Research methodology}

The case study analyses the official statistical data (from the Statistical Yearbook of Romania), registered for the period 2008 - 2017 (the post-accession period of Romania to the European Union). 
The general objective of the study is to highlight the evolution of wage differences in the public and private sector, depending on the order of size of enterprises, by number of employees and by gender (male / female).

Hypothesis 1 - Wage incomes in public enterprises are higher than those in the private sector, the gaps widening over time.

Hypothesis 2 - Due to economies of scale and productivity increases, wages in large enterprises (over 250 employees) are higher than in SMEs. Wage differences at the level of large enterprises are insignificant between the public and private sectors.

Hypothesis 3 - Gender discrimination from the perspective of salary income is registered in the case of all types of enterprises.

Hypothesis 4 - The economic dimension of sustainability is determined by a priority contribution of the private sector to the generation of surplus value, and therefore a primary distribution of revenues with priority to this sector.

In order to reach the proposed objectives and to test the hypotheses of the study, data from official statistics were used, regarding the level of net earnings obtained by employees in Romania, respectively the number of employees in Romanian enterprises. The data were collected from the 2009-2019 editions of the Statistical Yearbook of Romania, for the time interval of one decade, mentioned above, being centralized in the form of time series, using for their processing the statistical software. The analysis was performed for each category of enterprises, organized according to the following criteria:

- C1 - size (less than 50 employees, 50-249 employees and over 250 employees);

- C2 - type of sector: public and private;

- C3 - gender of employees (male and female).

For the correlation analysis of these data we proceeded to study the evolution over time, following a comparison between the categories of enterprises according to the above criteria, testing the research hypotheses using a simple unifactorial regression model, in order to establish the link between the two mentioned indicators. A time series represents a sequence of values recorded by a specific random variable in a time interval, being analysed the frequency of the series, therefore the periodicity with which the variable is observed. In this case, the frequency is annual. For the in-depth study of the evolution of the aforementioned indicators, one can analyse the oscillation over time of the values, through graphical representations, comparing successively the levels of two consecutive years. In order to verify the validity of the data used, as well as the level of representativeness, various statistical tests were taken into account, the results obtained being interpreted in the content of the present case study.

\subsection{Data analysis}

The input data used in the study were centralized in the following tables:

Table 1: Average monthly net nominal earnings, by size classes of enterprises and gender

\begin{tabular}{|c|c|c|c|c|c|c|c|c|c|c|c|c|}
\hline & & & $\begin{array}{l}200 \\
8\end{array}$ & $\begin{array}{l}200 \\
9\end{array}$ & $\begin{array}{l}201 \\
0\end{array}$ & $\begin{array}{l}201 \\
1\end{array}$ & $\begin{array}{l}201 \\
2\end{array}$ & $\begin{array}{l}201 \\
3\end{array}$ & $\begin{array}{l}201 \\
4\end{array}$ & $\begin{array}{l}201 \\
5\end{array}$ & $\begin{array}{l}201 \\
6\end{array}$ & $\begin{array}{l}201 \\
7\end{array}$ \\
\hline \multirow{5}{*}{ 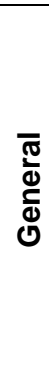 } & \multirow{2}{*}{$\begin{array}{l}\text { Under } 50 \\
\text { employe } \\
\text { es }\end{array}$} & Lei & 843 & 844 & 903 & 957 & 960 & 973 & $\begin{array}{r}108 \\
3 \\
\end{array}$ & $\begin{array}{r}123 \\
5 \\
\end{array}$ & $\begin{array}{r}136 \\
2 \\
\end{array}$ & $\begin{array}{r}160 \\
2 \\
\end{array}$ \\
\hline & & $\begin{array}{l}\text { eu } \\
\text { ro }\end{array}$ & $\begin{array}{r}228 . \\
90\end{array}$ & $\begin{array}{r}199 . \\
18\end{array}$ & $\begin{array}{r}214 . \\
49\end{array}$ & $\begin{array}{r}225 . \\
82\end{array}$ & $\begin{array}{r}215 . \\
44\end{array}$ & $\begin{array}{r}220 . \\
19\end{array}$ & $\begin{array}{r}243 . \\
67\end{array}$ & $\begin{array}{r}277 . \\
84\end{array}$ & $\begin{array}{r}303 . \\
29\end{array}$ & $\begin{array}{r}350 . \\
69\end{array}$ \\
\hline & \multirow{2}{*}{$\begin{array}{l}50-249 \\
\text { employe } \\
\text { es }\end{array}$} & Lei & $\begin{array}{r}121 \\
7\end{array}$ & $\begin{array}{r}129 \\
4\end{array}$ & $\begin{array}{r}135 \\
4\end{array}$ & $\begin{array}{r}138 \\
0\end{array}$ & $\begin{array}{r}143 \\
9\end{array}$ & $\begin{array}{r}151 \\
2\end{array}$ & $\begin{array}{r}161 \\
7\end{array}$ & $\begin{array}{r}179 \\
9\end{array}$ & $\begin{array}{r}205 \\
7\end{array}$ & $\begin{array}{r}235 \\
5\end{array}$ \\
\hline & & $\begin{array}{l}\text { eu } \\
\text { ro }\end{array}$ & $\begin{array}{r}330 . \\
46\end{array}$ & $\begin{array}{r}305 . \\
38\end{array}$ & $\begin{array}{r}321 . \\
62\end{array}$ & $\begin{array}{r}325 . \\
63\end{array}$ & $\begin{array}{r}322 . \\
94\end{array}$ & $\begin{array}{r}342 . \\
16\end{array}$ & $\begin{array}{r}363 . \\
81\end{array}$ & $\begin{array}{r}404 . \\
72\end{array}$ & $\begin{array}{r}458 . \\
05\end{array}$ & $\begin{array}{r}515 . \\
53\end{array}$ \\
\hline & $\begin{array}{l}\text { Over } 250 \\
\text { employe }\end{array}$ & Iei & $\begin{array}{r}164 \\
4\end{array}$ & $\begin{array}{r}173 \\
5\end{array}$ & $\begin{array}{r}171 \\
1\end{array}$ & $\begin{array}{r}175 \\
9\end{array}$ & $\begin{array}{r}185 \\
4\end{array}$ & $\begin{array}{r}198 \\
8\end{array}$ & $\begin{array}{r}213 \\
0\end{array}$ & $\begin{array}{r}229 \\
6\end{array}$ & $\begin{array}{r}251 \\
9\end{array}$ & $\begin{array}{r}287 \\
3\end{array}$ \\
\hline
\end{tabular}




\begin{tabular}{|c|c|c|c|c|c|c|c|c|c|c|c|c|}
\hline & es & $\begin{array}{l}\text { eu } \\
\text { ro }\end{array}$ & $\begin{array}{r}446 . \\
41\end{array}$ & $\begin{array}{r}409 . \\
46\end{array}$ & $\begin{array}{r}406 . \\
42\end{array}$ & $\begin{array}{r}415 . \\
06\end{array}$ & $\begin{array}{r}416 . \\
07\end{array}$ & $\begin{array}{r}449 . \\
88\end{array}$ & $\begin{array}{r}479 . \\
23\end{array}$ & $\begin{array}{r}516 . \\
54\end{array}$ & $\begin{array}{r}560 . \\
93\end{array}$ & $\begin{array}{r}628 . \\
93\end{array}$ \\
\hline \multirow{11}{*}{ 选 } & \multirow{3}{*}{$\begin{array}{l}\text { Under } 50 \\
\text { employe } \\
\text { es }\end{array}$} & lei & 106 & 107 & 117 & 123 & 125 & 129 & 145 & 161 & 179 & 214 \\
\hline & & & 7 & 5 & 0 & 3 & 9 & 7 & 4 & 4 & 2 & 8 \\
\hline & & eu & 289. & 253. & 277. & $\begin{array}{r}290 . \\
95\end{array}$ & 282. & 293. & $\begin{array}{r}327 . \\
\end{array}$ & 363. & 399. & 470. \\
\hline & \multirow{4}{*}{$\begin{array}{l}50-249 \\
\text { employe } \\
\text { es }\end{array}$} & lei & 157 & 168 & 174 & 178 & 189 & 200 & 215 & 241 & 272 & 317 \\
\hline & & & 9 & 6 & 2 & 6 & 7 & 5 & 1 & 1 & 3 & 1 \\
\hline & & eu & 428. & 397. & 413. & 421. & 425. & 453. & 483. & 542. & 606. & 694. \\
\hline & & ro & 76 & 90 & 88 & 44 & 72 & 72 & 96 & 41 & 35 & 16 \\
\hline & \multirow{4}{*}{$\begin{array}{l}\text { Over } 250 \\
\text { employe } \\
\text { es }\end{array}$} & lei & 209 & 221 & 214 & 219 & 232 & 250 & 268 & 292 & 322 & 375 \\
\hline & & & 3 & 4 & 5 & 1 & 2 & 2 & 7 & 7 & 5 & 0 \\
\hline & & eu & 568. & 522. & 509. & 517. & 521. & 566. & 604. & 658. & 718. & 820. \\
\hline & & ro & 33 & 50 & 51 & 00 & 10 & 19 & 55 & 49 & 13 & 91 \\
\hline \multirow{12}{*}{$\frac{\frac{0}{\pi}}{\Sigma}$} & \multirow{4}{*}{$\begin{array}{l}\text { Under } 50 \\
\text { employe } \\
\text { es }\end{array}$} & lei & 116 & 118 & 127 & 136 & 133 & 134 & 149 & 174 & 191 & 223 \\
\hline & & & 7 & 3 & 2 & 3 & 7 & 4 & 8 & 9 & 6 & 4 \\
\hline & & $e u$ & 316. & 279. & 302. & 321. & 300. & 304. & 337. & 393. & 426. & 489. \\
\hline & & ro & 89 & 19 & 14 & 62 & 04 & 14 & 04 & 48 & 65 & 04 \\
\hline & \multirow{4}{*}{$\begin{array}{l}50-249 \\
\text { employe } \\
\text { es }\end{array}$} & lei & 166 & 179 & 192 & 195 & 201 & 211 & 226 & 251 & 288 & 325 \\
\hline & & & 4 & 2 & 4 & 8 & 2 & 1 & 5 & 0 & 7 & 7 \\
\hline & & eu & 451. & 422. & 457. & 462. & 451. & 477. & 509. & 564. & 642. & 712. \\
\hline & & ro & 84 & 91 & 02 & 02 & 52 & 71 & 61 & 68 & 87 & 99 \\
\hline & \multirow{4}{*}{$\begin{array}{l}\text { Over } 250 \\
\text { employe } \\
\text { es }\end{array}$} & lei & 235 & 251 & 255 & 265 & 278 & 297 & 318 & 341 & 373 & 420 \\
\hline & & & 2 & 4 & 9 & 0 & 7 & 7 & 2 & 7 & 2 & 3 \\
\hline & & eu & 638. & 593. & 607. & 625. & 625. & 673. & 715. & 768. & 831. & 920. \\
\hline & & ro & 66 & 30 & 85 & 31 & 45 & 68 & 92 & 73 & 03 & 08 \\
\hline
\end{tabular}

Source: authors' processing, apud the information from Romania's Statistic Yearbook, 2018

With regard to the average monthly net nominal earnings, there is a steady increase, but not equal between two consecutive years, in all categories of wages, highlighting the differences in income between the category of small and medium enterprises and those with more than 250 employees. The ratio between the level of net female and male nominal earnings highlights the lack of homogeneity over time and by size classes of enterprises.

Table 2: Differences and salary ratio male - female

\begin{tabular}{|c|c|c|c|c|c|c|c|c|c|}
\hline \multirow[t]{3}{*}{ Year } & \multicolumn{6}{|c|}{ Male - female salary differences } & \multicolumn{3}{|c|}{ Female - male salary ratio (\%) } \\
\hline & \multicolumn{2}{|c|}{$\begin{array}{c}\text { Under } 50 \\
\text { employees }\end{array}$} & \multicolumn{2}{|c|}{$\begin{array}{c}50-249 \\
\text { employees }\end{array}$} & \multicolumn{2}{|c|}{$\begin{array}{c}\text { Over } 250 \\
\text { employees }\end{array}$} & \multirow[t]{2}{*}{$\begin{array}{c}\text { Under } 50 \\
\text { employees }\end{array}$} & \multirow[t]{2}{*}{$\begin{array}{c}50-249 \\
\text { employees }\end{array}$} & \multirow[t]{2}{*}{$\begin{array}{c}\text { Over } 250 \\
\text { employees }\end{array}$} \\
\hline & lei & euro & Lei & euro & Lei & Euro & & & \\
\hline 2008 & 100 & 27.15 & 85 & 23.08 & 259 & 70.33 & 0.9143 & 0.9489 & 0.8899 \\
\hline 2009 & 108 & 25.49 & 106 & 25.02 & 300 & 70.80 & 0.9087 & 408 & 0.8807 \\
\hline 2010 & 102 & 24.23 & 182 & 43.23 & 414 & 98.34 & 0.9198 & 054 & 0.8382 \\
\hline 2011 & 130 & 30.68 & 172 & 40.59 & 459 & 108.31 & 0.9046 & 122 & 0.8268 \\
\hline 2012 & 78 & 17.50 & 115 & 25.81 & 465 & 104.35 & 0.9417 & 428 & 0.8332 \\
\hline 2013 & 47 & 10.64 & 106 & 23.99 & 475 & 107.49 & 0.9650 & 498 & 0.8404 \\
\hline 2014 & 44 & 9.90 & 114 & 25.65 & 495 & 111.37 & 0.9706 & 0.9497 & 0.8444 \\
\hline 2015 & 135 & 30.37 & 99 & 22.72 & 490 & 110.24 & 0.9228 & 0.9606 & 0.8566 \\
\hline 2016 & 124 & 27.61 & 164 & 36.52 & 507 & 112.90 & 0.9353 & 0.9432 & 0.8641 \\
\hline 2017 & 86 & 18.83 & 86 & 18.83 & 453 & 99.17 & 0.9615 & 0.9736 & 0.8922 \\
\hline
\end{tabular}

Source: authors' processing

The gender pay gap is favourable for men, for the whole period analysed, for all three categories of enterprises, the ratio between nominal female / male earnings being between $90.46 \%$ in 2011 and $97.06 \%$ in 2014, the period of the ten years analysed recording variations of this report. The calculated values are lower in terms of enterprises with more 
than 250 employees, where the lowest level of the report is recorded in $2011,82.68 \%$, the highest level corresponding to $2017-89.22 \%$. On the average, during the ten years analysed, the ratio of the number of female / male employees is $78.35 \%$ for companies with less than 50 employees, $74.54 \%$ for those with $50-249$ employees and $105.17 \%$ for those with more than 250 employees.

Table 3: Net nominal average wage gaps (euro), by companies' size classes

\begin{tabular}{|c|c|c|c|c|c|c|c|c|c|}
\hline Company & $\Delta_{\frac{2009}{2008}}$ & $\Delta_{\frac{2010}{2009}}$ & $\Delta_{\frac{2011}{2010}}$ & $\Delta_{\frac{2012}{2011}}$ & $\Delta_{\frac{2013}{2012}}$ & $\Delta_{\frac{2014}{2013}}$ & $\Delta_{\frac{2015}{2014}}$ & $\frac{\Delta_{2016}}{2015}$ & $\Delta_{\frac{2017}{2016}}$ \\
\hline $\begin{array}{c}\text { Under } 50 \\
\text { employe } \\
\text { es }\end{array}$ & $\begin{array}{c}87.0 \\
2 \%\end{array}$ & $\begin{array}{c}107.6 \\
9 \%\end{array}$ & $\begin{array}{c}105.2 \\
8 \%\end{array}$ & $\begin{array}{c}95.56 \\
\%\end{array}$ & $\begin{array}{c}102.2 \\
0 \%\end{array}$ & $\begin{array}{c}110.6 \\
6 \%\end{array}$ & $\begin{array}{c}114.0 \\
1 \%\end{array}$ & $\begin{array}{c}109.1 \\
6 \%\end{array}$ & $\begin{array}{c}100.7 \\
9 \%\end{array}$ \\
\hline $\begin{array}{c}50-249 \\
\text { employe } \\
\text { es }\end{array}$ & $\begin{array}{c}92.4 \\
1 \%\end{array}$ & $\begin{array}{c}105.3 \\
2 \%\end{array}$ & $\begin{array}{c}101.2 \\
5 \%\end{array}$ & $\begin{array}{c}99.17 \\
\%\end{array}$ & $\begin{array}{c}105.9 \\
5 \%\end{array}$ & $\begin{array}{c}106.3 \\
3 \%\end{array}$ & $\begin{array}{c}111.2 \\
5 \%\end{array}$ & $\begin{array}{c}113.1 \\
8 \%\end{array}$ & $\begin{array}{c}112.5 \\
5 \%\end{array}$ \\
\hline $\begin{array}{c}\text { Over } 250 \\
\text { employe } \\
\text { es }\end{array}$ & $\begin{array}{c}91.7 \\
2 \%\end{array}$ & $\begin{array}{c}99.26 \\
\%\end{array}$ & $\begin{array}{c}102.1 \\
2 \%\end{array}$ & $\begin{array}{c}100.2 \\
4 \%\end{array}$ & $\begin{array}{c}108.1 \\
2 \%\end{array}$ & $\begin{array}{c}99.86 \\
\%\end{array}$ & $\begin{array}{c}107.7 \\
9 \%\end{array}$ & $\begin{array}{c}108.5 \\
9 \%\end{array}$ & $\begin{array}{c}112.1 \\
2 \%\end{array}$ \\
\hline
\end{tabular}

Source: authors' processing

In 2009 compared to 2008, during the economic crisis, wages decreased, affecting mostly small enterprises (with less than 50 employees), the situation being remedied slowly, except for 2012, when, compared to 2011, at in this category and in medium-sized enterprises, there is a small decrease, and in large enterprises, the income situation deteriorates slightly in 2014 compared to 2015.

Small businesses are those that, although dominant in number in the economy, do not find solutions to increase revenue, especially due to low productivity and a poor level of technical equipment. Large companies, with over 250 employees, although the least numerous, have a favourable evolution of salary incomes, mainly due to the efficiency of management and know-how implemented in subsidiaries of multinational companies.

Table 4: Average monthly net nominal earnings, by size classes of companies and by gender, for the public sector

\begin{tabular}{|c|c|c|c|c|c|c|c|c|c|c|c|c|}
\hline \multicolumn{2}{|c|}{$\begin{array}{l}\text { Company } \\
\text { category }\end{array}$} & m.u & 2008 & 2009 & 2010 & 2011 & 2012 & 2013 & 2014 & 2015 & 2016 & 2017 \\
\hline \multirow{6}{*}{$\begin{array}{l}\bar{\sigma} \\
\frac{\pi}{\varpi} \\
\mathbb{\Phi}\end{array}$} & Under & Lei & 1503 & 1662 & 1448 & 1402 & 1519 & 1689 & 1792 & 1930 & 2237 & 2858 \\
\hline & $\begin{array}{l}50 \\
\text { employe } \\
\text { es }\end{array}$ & $\begin{array}{l}\text { Eur } \\
0\end{array}$ & $\begin{array}{r}408 . \\
12\end{array}$ & $\begin{array}{r}392 . \\
23\end{array}$ & $\begin{array}{r}343 . \\
95\end{array}$ & $\begin{array}{r}330 . \\
85\end{array}$ & $\begin{array}{r}340 . \\
89\end{array}$ & $\begin{array}{r}382 . \\
21\end{array}$ & $\begin{array}{r}403 . \\
19\end{array}$ & $\begin{array}{r}434 . \\
20\end{array}$ & $\begin{array}{r}498 . \\
13\end{array}$ & $\begin{array}{r}625 . \\
64\end{array}$ \\
\hline & $50-249$ & Lei & 2229 & 2107 & 1983 & 1994 & 2186 & 2377 & 2609 & 2807 & 3128 & 3969 \\
\hline & $\begin{array}{l}\text { employe } \\
\text { es }\end{array}$ & $\begin{array}{l}\text { Eur } \\
\text { o }\end{array}$ & $\begin{array}{r}605 . \\
26\end{array}$ & $\begin{array}{r}497 . \\
25\end{array}$ & $\begin{array}{r}471 . \\
03\end{array}$ & $\begin{array}{r}470 . \\
52\end{array}$ & $\begin{array}{r}490 . \\
57\end{array}$ & $\begin{array}{r}537 . \\
90\end{array}$ & $\begin{array}{r}587 . \\
00\end{array}$ & $\begin{array}{r}631 . \\
50\end{array}$ & $\begin{array}{r}696 . \\
54\end{array}$ & $\begin{array}{r}868 . \\
85\end{array}$ \\
\hline & Over & Lei & 2433 & 2458 & 2318 & 2308 & 2429 & 2639 & 2859 & 3068 & 3398 & 4048 \\
\hline & $\begin{array}{l}250 \\
\text { employe } \\
\text { es }\end{array}$ & $\begin{array}{l}\text { Eur } \\
\text { o }\end{array}$ & $\begin{array}{r}660 . \\
66\end{array}$ & $\begin{array}{r}580 . \\
09\end{array}$ & $\begin{array}{r}550 . \\
61\end{array}$ & $\begin{array}{r}544 . \\
61\end{array}$ & $\begin{array}{r}545 . \\
11\end{array}$ & $\begin{array}{r}597 . \\
19\end{array}$ & $\begin{array}{r}643 . \\
25\end{array}$ & $\begin{array}{r}690 . \\
21\end{array}$ & $\begin{array}{r}756 . \\
66\end{array}$ & $\begin{array}{r}886 . \\
15\end{array}$ \\
\hline \multirow{6}{*}{ 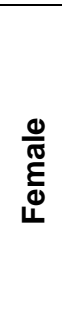 } & Under & Lei & 1395 & 1544 & 1352 & 1326 & 1464 & 1622 & 1724 & 1887 & 2183 & 2749 \\
\hline & $\begin{array}{l}50 \\
\text { employe } \\
\text { es }\end{array}$ & $\begin{array}{l}\text { Eur } \\
\text { o }\end{array}$ & $\begin{array}{r}378 . \\
80\end{array}$ & $\begin{array}{r}364 . \\
38\end{array}$ & $\begin{array}{r}321 . \\
15\end{array}$ & $\begin{array}{r}312 . \\
89\end{array}$ & $\begin{array}{r}328 . \\
55\end{array}$ & $\begin{array}{r}367 . \\
05\end{array}$ & $\begin{array}{r}387 . \\
89\end{array}$ & $\begin{array}{r}424 . \\
52\end{array}$ & $\begin{array}{r}486 . \\
10\end{array}$ & $\begin{array}{r}601 . \\
78\end{array}$ \\
\hline & $50-249$ & Lei & 2293 & 2086 & 1977 & 1969 & 2178 & 2384 & 2629 & 2823 & 3147 & 4029 \\
\hline & $\begin{array}{l}\text { employe } \\
\text { es }\end{array}$ & $\begin{array}{l}\text { Eur } \\
0\end{array}$ & $\begin{array}{r}622 . \\
64\end{array}$ & $\begin{array}{r}492 . \\
29\end{array}$ & $\begin{array}{r}469 . \\
61\end{array}$ & $\begin{array}{r}464 . \\
62\end{array}$ & $\begin{array}{r}488 . \\
78\end{array}$ & $\begin{array}{r}539 . \\
49\end{array}$ & $\begin{array}{r}591 . \\
50\end{array}$ & $\begin{array}{r}635 . \\
10\end{array}$ & $\begin{array}{r}700 . \\
77\end{array}$ & $\begin{array}{r}881 . \\
99\end{array}$ \\
\hline & Over & Lei & 2261 & 2269 & 2078 & 2028 & 2164 & 2396 & 2635 & 2854 & 3191 & 3897 \\
\hline & 250 & Eur & 613. & 535. & 493. & 478. & 485. & 542. & 592. & 642. & 710. & 853. \\
\hline
\end{tabular}


The Annals of the University of Oradea. Economic Sciences

TOM XXX, $1^{\text {st }}$ Issue, July 2021

\begin{tabular}{|c|c|c|c|c|c|c|c|c|c|c|c|c|}
\hline & $\begin{array}{l}\text { employe } \\
\text { es }\end{array}$ & 0 & 95 & 48 & 60 & 54 & 64 & 20 & 85 & 07 & 56 & 09 \\
\hline \multirow{6}{*}{$\frac{0}{\frac{0}{\pi}}$} & \multirow{2}{*}{$\begin{array}{l}\text { Under } \\
50 \\
\text { employe } \\
\text { es }\end{array}$} & Lei & 1637 & 1805 & 1568 & 1496 & 1585 & 1767 & 1871 & 1984 & 2305 & 2993 \\
\hline & & $\begin{array}{l}\text { Eur } \\
0\end{array}$ & $\begin{array}{r}444 . \\
51\end{array}$ & $\begin{array}{r}425 . \\
98\end{array}$ & $\begin{array}{r}372 . \\
46\end{array}$ & $\begin{array}{r}353 . \\
00\end{array}$ & $\begin{array}{r}355 . \\
70\end{array}$ & $\begin{array}{r}399 . \\
86\end{array}$ & $\begin{array}{r}420 . \\
96\end{array}$ & $\begin{array}{r}446 . \\
34\end{array}$ & $\begin{array}{r}513 . \\
27\end{array}$ & $\begin{array}{r}655 . \\
20\end{array}$ \\
\hline & \multirow{2}{*}{$\begin{array}{l}50-249 \\
\text { employe } \\
\text { es }\end{array}$} & Lei & 2163 & 2132 & 1998 & 2027 & 2194 & 2369 & 2583 & 2787 & 3104 & 3727 \\
\hline & & $\begin{array}{l}\text { Eur } \\
\text { o }\end{array}$ & $\begin{array}{r}587 . \\
34\end{array}$ & $\begin{array}{r}503 . \\
15\end{array}$ & $\begin{array}{r}474 . \\
60\end{array}$ & $\begin{array}{r}478 . \\
30\end{array}$ & $\begin{array}{r}492 . \\
37\end{array}$ & $\begin{array}{r}536 . \\
09\end{array}$ & $\begin{array}{r}581 . \\
15\end{array}$ & $\begin{array}{r}627 . \\
00\end{array}$ & $\begin{array}{r}691 . \\
19\end{array}$ & $\begin{array}{r}815 . \\
88\end{array}$ \\
\hline & \multirow{2}{*}{$\begin{array}{l}\text { Over } \\
250 \\
\text { employe } \\
\text { es }\end{array}$} & Lei & 2667 & 2723 & 2650 & 2696 & 2805 & 2995 & 3192 & 3394 & 3727 & 4295 \\
\hline & & $\begin{array}{l}\text { Eur } \\
0\end{array}$ & $\begin{array}{r}724 . \\
20\end{array}$ & $\begin{array}{r}642 . \\
63\end{array}$ & $\begin{array}{r}629 . \\
47\end{array}$ & $\begin{array}{r}636 . \\
16\end{array}$ & $\begin{array}{r}629 . \\
49\end{array}$ & $\begin{array}{r}677 . \\
76\end{array}$ & $\begin{array}{r}718 . \\
17\end{array}$ & $\begin{array}{r}763 . \\
55\end{array}$ & $\begin{array}{r}829 . \\
92\end{array}$ & $\begin{array}{r}940 . \\
22\end{array}$ \\
\hline
\end{tabular}

Source: authors' processing, apud the information from Romania's Statistic Yearbook, 2018

In the public sector, due to the austerity measures taken by the Romanian Government to reduce the effects of the economic crisis, there was a reduction in the level of wages in the budget sector by $25 \%$ in $2010-2011$, the level of average monthly net earnings fluctuated and major increases were recorded in the last two years analysed.

Gender pay gaps are favourable for men, for public enterprises with less than 50 and over 250 employees, the ratio between nominal female / male earnings being between $85.22 \%$ in 2008 and $95.11 \%$ in 2015, in the analysed interval there are variations of this ratio. The exception to this trend is the value of the average net monthly nominal earnings for public enterprises with 50-249 employees, where the salary level of women exceeds that of men constantly, since 2013.

Table 5: Differences and male - female wage ratio in the public sector

\begin{tabular}{|c|c|c|c|c|c|c|c|c|c|}
\hline \multirow[t]{3}{*}{ Year } & \multicolumn{6}{|c|}{ Male - female salary differences (lei) } & \multicolumn{3}{|c|}{ Female - male salary ratio (\%) } \\
\hline & \multicolumn{2}{|c|}{$\begin{array}{c}\text { Under } 50 \\
\text { employees }\end{array}$} & \multicolumn{2}{|c|}{$\begin{array}{c}50-249 \\
\text { employees }\end{array}$} & \multicolumn{2}{|c|}{$\begin{array}{c}\text { Over } 250 \\
\text { employees }\end{array}$} & \multirow[t]{2}{*}{$\begin{array}{c}\text { Under } 50 \\
\text { employees }\end{array}$} & \multirow[t]{2}{*}{$\begin{array}{c}50-249 \\
\text { employees }\end{array}$} & \multirow[t]{2}{*}{$\begin{array}{l}\text { Over } 250 \\
\text { employees }\end{array}$} \\
\hline & Lei & euro & Lei & euro & Lei & euro & & & \\
\hline 2008 & 242 & 65.71 & -130 & 35.30 & 405 & 109.97 & 0.8522 & 1.0601 & 0.8481 \\
\hline 2009 & 261 & 61.60 & 46 & 10.86 & 454 & 107.14 & 0.8554 & 0.9784 & 0.8333 \\
\hline 2010 & 216 & 51.31 & 21 & 4.99 & 572 & 135.87 & 0.8622 & 895 & .7842 \\
\hline 2011 & 170 & 40.11 & 58 & 13.69 & 668 & 157.63 & 0.8864 & 9714 & 0.7522 \\
\hline 2012 & 121 & 27.15 & 16 & 3.59 & 641 & 143.85 & 237 & 927 & 0.7715 \\
\hline 2013 & 145 & 32.81 & -15 & -3.39 & 599 & 135.55 & 179 & 063 & 0.8000 \\
\hline 2014 & 147 & 33.07 & -46 & 10.35 & 557 & 125.32 & 0.9214 & 1.0178 & 0.8255 \\
\hline 2015 & 97 & 21.82 & -36 & -8.10 & 540 & 121.48 & 511 & 129 & 0.8409 \\
\hline 2016 & 122 & 27.17 & -42 & -9.35 & 536 & 119.36 & 0.9471 & 135 & 0.8562 \\
\hline 2017 & 244 & 53.41 & -137 & 29.99 & 398 & 87.13 & 0.9185 & 1.0352 & 0.9073 \\
\hline
\end{tabular}

Source: authors' processing, apud the information from Romania's Statistic Yearbook, 2018

On average, during the ten years analysed, the ratio of the number of female / male employees is $121.35 \%$ for public enterprises with less than 50 employees, $125.18 \%$ for those with $50-249$ employees and $146.70 \%$ for those with over 250 employees, highlighting the more pronounced female character of the organizational culture in the enterprises subordinated to the public power. 
Table 6: Average monthly net nominal earnings, by size classes of enterprises and by sex, for the private sector

\begin{tabular}{|c|c|c|c|c|c|c|c|c|c|c|c|c|}
\hline \multicolumn{2}{|c|}{$\begin{array}{l}\text { Company } \\
\text { category }\end{array}$} & $\begin{array}{l}\mathrm{m} . \\
\mathrm{u} .\end{array}$ & 2008 & 2009 & 2010 & 2011 & 2012 & 2013 & 2014 & 2015 & 2016 & 2017 \\
\hline \multirow{6}{*}{$\begin{array}{l}\bar{\sigma} \\
\frac{\pi}{0} \\
\bar{\Phi} \\
\mathbb{0}\end{array}$} & \multirow{2}{*}{$\begin{array}{l}\text { Over } 50 \\
\text { employe } \\
\text { es }\end{array}$} & lei & 1100 & 1096 & 1205 & 1294 & 1280 & 1288 & 1449 & 1669 & 1833 & 2148 \\
\hline & & $\begin{array}{l}\text { eur } \\
0\end{array}$ & $\begin{array}{l}298 . \\
69\end{array}$ & $\begin{array}{l}258 . \\
66\end{array}$ & $\begin{array}{l}286 . \\
23\end{array}$ & $\begin{array}{l}305 . \\
34\end{array}$ & $\begin{array}{l}287 . \\
25\end{array}$ & $\begin{array}{l}291 . \\
47\end{array}$ & $\begin{array}{l}326 . \\
01\end{array}$ & $\begin{array}{l}375 . \\
48\end{array}$ & $\begin{array}{l}408 . \\
17\end{array}$ & $\begin{array}{l}470 . \\
22\end{array}$ \\
\hline & \multirow{2}{*}{$\begin{array}{l}50-249 \\
\text { employe } \\
\text { es }\end{array}$} & lei & 1503 & 1646 & 1804 & 1860 & 1921 & 2004 & 2136 & 2402 & 2751 & 3050 \\
\hline & & $\begin{array}{l}\text { eur } \\
0\end{array}$ & $\begin{array}{l}408 . \\
12\end{array}$ & $\begin{array}{l}388 . \\
45\end{array}$ & $\begin{array}{l}428 . \\
51\end{array}$ & $\begin{array}{l}438 . \\
90\end{array}$ & $\begin{array}{l}431 . \\
10\end{array}$ & $\begin{array}{l}453 . \\
50\end{array}$ & $\begin{array}{l}480 . \\
58\end{array}$ & $\begin{array}{l}540 . \\
38\end{array}$ & $\begin{array}{l}612 . \\
59\end{array}$ & $\begin{array}{l}667 . \\
67\end{array}$ \\
\hline & \multirow{2}{*}{$\begin{array}{l}\text { Over } \\
250 \\
\text { employe } \\
\text { es }\end{array}$} & lei & 2026 & 2264 & 2380 & 2519 & 2658 & 2813 & 2982 & 3237 & 3517 & 3909 \\
\hline & & $\begin{array}{l}\text { eur } \\
0\end{array}$ & $\begin{array}{l}550 . \\
14\end{array}$ & $\begin{array}{l}534 . \\
30\end{array}$ & $\begin{array}{l}565 . \\
33\end{array}$ & $\begin{array}{l}594 . \\
40\end{array}$ & $\begin{array}{l}596 . \\
50\end{array}$ & $\begin{array}{l}636 . \\
57\end{array}$ & $\begin{array}{l}670 . \\
93\end{array}$ & $\begin{array}{l}728 . \\
23\end{array}$ & $\begin{array}{l}783 . \\
16\end{array}$ & $\begin{array}{l}855 . \\
72\end{array}$ \\
\hline \multirow{6}{*}{$\begin{array}{l}\frac{0}{\pi} \\
\frac{\pi}{0} \\
\frac{0}{4}\end{array}$} & \multirow{2}{*}{$\begin{array}{l}\text { Under } \\
50 \\
\text { employe } \\
\text { es }\end{array}$} & lei & 781 & 767 & 846 & 896 & 910 & 924 & 1043 & & & 1526 \\
\hline & & $\begin{array}{l}\text { eur } \\
\text { o }\end{array}$ & $\begin{array}{l}213 . \\
81\end{array}$ & $\begin{array}{l}181 . \\
01\end{array}$ & $\begin{array}{l}200 . \\
95\end{array}$ & $\begin{array}{l}211 . \\
43\end{array}$ & $\begin{array}{l}204 . \\
22\end{array}$ & $\begin{array}{l}209 . \\
10\end{array}$ & $\begin{array}{l}234 . \\
67\end{array}$ & $\begin{array}{l}260 . \\
52\end{array}$ & $\begin{array}{l}285 . \\
47\end{array}$ & $\begin{array}{l}334 . \\
06\end{array}$ \\
\hline & \multirow{2}{*}{$\begin{array}{l}50-249 \\
\text { employe } \\
\text { es }\end{array}$} & lei & 1043 & 1145 & 1220 & 269 & & 1394 & 1478 & & & 2108 \\
\hline & & $\begin{array}{l}\text { eur } \\
0\end{array}$ & $\begin{array}{l}283 . \\
22\end{array}$ & $\begin{array}{l}270 . \\
22\end{array}$ & $\begin{array}{l}289 . \\
79\end{array}$ & $\begin{array}{l}299 . \\
44\end{array}$ & $\begin{array}{l}300 . \\
27\end{array}$ & $\begin{array}{l}315 . \\
46\end{array}$ & $\begin{array}{l}333 . \\
89\end{array}$ & $\begin{array}{l}377 . \\
05\end{array}$ & $\begin{array}{l}423 . \\
31\end{array}$ & $\begin{array}{l}461 . \\
46\end{array}$ \\
\hline & \multirow{2}{*}{$\begin{array}{l}\text { Over } \\
250 \\
\text { employe } \\
\text { es }\end{array}$} & lei & 1401 & 1581 & 1635 & 1745 & 1833 & 1919 & 2002 & 2187 & 2382 & 2635 \\
\hline & & $\begin{array}{l}\text { eur } \\
\text { o }\end{array}$ & $\begin{array}{l}380 . \\
43\end{array}$ & $\begin{array}{l}373 . \\
11\end{array}$ & $\begin{array}{l}388 . \\
37\end{array}$ & $\begin{array}{l}411 . \\
76\end{array}$ & $\begin{array}{l}411 . \\
36\end{array}$ & $\begin{array}{l}434 . \\
26\end{array}$ & $\begin{array}{l}450 . \\
43\end{array}$ & $\begin{array}{l}492 . \\
01\end{array}$ & $\begin{array}{l}530 . \\
42\end{array}$ & $\begin{array}{l}576 . \\
83\end{array}$ \\
\hline \multirow{6}{*}{$\frac{0}{\Sigma}$} & \multirow{2}{*}{$\begin{array}{l}\text { Under } \\
50 \\
\text { employe } \\
\text { es }\end{array}$} & lei & 859 & 852 & 920 & & 971 & 964 & 1077 & & & 1600 \\
\hline & & $\begin{array}{l}\text { eur } \\
0\end{array}$ & $\begin{array}{l}255 . \\
45\end{array}$ & $\begin{array}{l}201 . \\
07\end{array}$ & $\begin{array}{l}218 . \\
53\end{array}$ & $\begin{array}{l}233 . \\
84\end{array}$ & $\begin{array}{l}217 . \\
91\end{array}$ & $\begin{array}{l}218 . \\
15\end{array}$ & $\begin{array}{l}242 . \\
32\end{array}$ & $\begin{array}{l}285 . \\
04\end{array}$ & $\begin{array}{l}308 . \\
19\end{array}$ & $\begin{array}{l}350 . \\
26\end{array}$ \\
\hline & \multirow{2}{*}{$\begin{array}{l}50-249 \\
\text { employe } \\
\text { es }\end{array}$} & lei & 1181 & 1275 & 1401 & 1429 & 1457 & 1520 & 1622 & & & 2294 \\
\hline & & $\begin{array}{l}\text { eur } \\
\text { o }\end{array}$ & $\begin{array}{l}320 . \\
69\end{array}$ & $\begin{array}{l}300 . \\
90\end{array}$ & $\begin{array}{l}332 . \\
79\end{array}$ & 337. & $\begin{array}{l}326 . \\
97\end{array}$ & $\begin{array}{l}343 . \\
97\end{array}$ & $\begin{array}{l}364 . \\
94\end{array}$ & $\begin{array}{l}405 . \\
85\end{array}$ & $\begin{array}{l}465 . \\
41\end{array}$ & $\begin{array}{l}502 . \\
18\end{array}$ \\
\hline & \multirow{2}{*}{$\begin{array}{l}\text { Over } \\
250 \\
\text { employe } \\
\text { es }\end{array}$} & lei & 1581 & 1738 & 1813 & 1907 & 2028 & 2170 & 2322 & 2503 & 2730 & 3027 \\
\hline & & $\begin{array}{l}\text { eur } \\
\text { o }\end{array}$ & $\begin{array}{l}429 . \\
30\end{array}$ & $\begin{array}{l}410 . \\
17\end{array}$ & $\begin{array}{l}430 . \\
65\end{array}$ & $\begin{array}{l}450 . \\
02\end{array}$ & $\begin{array}{l}455 . \\
12\end{array}$ & $\begin{array}{l}491 . \\
06\end{array}$ & $\begin{array}{l}522 . \\
43\end{array}$ & $\begin{array}{l}563 . \\
10\end{array}$ & $\begin{array}{l}607 . \\
91\end{array}$ & $\begin{array}{l}662 . \\
64\end{array}$ \\
\hline
\end{tabular}

Source: authors' processing, apud the information from Romania's Statistic Yearbook, 2018

As regards the private sector, there is a greater gap between wage levels by size groups of enterprises, a steady but slower evolution of the level of wages for enterprises under 50 employees, due to increases in the minimum wage imposed by the state and low labour productivity. For enterprises with 50-249 employees, the female-male differences are significant, and for enterprises with more than 250 employees, the differences from the public sector are smaller than from the other two categories of enterprises. The male-female wage differences are constantly favourable to the male gender, regardless of the size of private enterprises, however, there are significant differences in 2017 in private enterprises with over 250 employees, in which the salaries obtained by women represent only $87.05 \%$ of those earned by men. 
Table 7: Differences and male - female wage ratio in the private sector

\begin{tabular}{|c|c|c|c|c|c|c|c|c|c|}
\hline \multirow[t]{3}{*}{ Years } & \multicolumn{6}{|c|}{ Male - female salary differences } & \multicolumn{3}{|c|}{ Female - male salary ratio (\%) } \\
\hline & \multicolumn{2}{|c|}{$\begin{array}{c}\text { Under } 50 \\
\text { employees }\end{array}$} & \multicolumn{2}{|c|}{$\begin{array}{c}50-249 \\
\text { employees }\end{array}$} & \multicolumn{2}{|c|}{$\begin{array}{c}\text { Over } 250 \\
\text { employees }\end{array}$} & \multirow[t]{2}{*}{$\begin{array}{c}\text { Under } 50 \\
\text { employees }\end{array}$} & \multirow[t]{2}{*}{$\begin{array}{c}50-249 \\
\text { employees }\end{array}$} & \multirow[t]{2}{*}{$\begin{array}{l}\text { Over } 250 \\
\text { employees }\end{array}$} \\
\hline & Lei & Euro & Lei & euro & Lei & euro & & & \\
\hline 2008 & 78 & 21.18 & 138 & 37.47 & 180 & 48.88 & 0.9092 & 0.8831 & 0.8861 \\
\hline 2009 & 85 & 20.06 & 130 & 30.68 & 157 & 37.05 & & & 097 \\
\hline 2010 & 74 & 17.58 & 181 & 42.99 & 178 & 42.28 & & 708 & 9018 \\
\hline 2011 & 95 & 22.42 & 160 & 37.75 & 162 & 38.23 & & & 9150 \\
\hline 2012 & 61 & 13.69 & 119 & 26.71 & 195 & 43.76 & & 183 & 0.9038 \\
\hline 2013 & 40 & 9.05 & 126 & 28.51 & 251 & 56.80 & & 9171 & 0.8873 \\
\hline 2014 & 34 & 7.65 & 144 & 32.40 & 320 & 72.00 & 684 & .9112 & 0.8622 \\
\hline 2015 & 109 & 24.52 & 128 & 28.80 & 316 & 71.09 & & .9290 & 0.8738 \\
\hline 2016 & 102 & 22.71 & 190 & 42.31 & 348 & 77.49 & & .9091 & 0.8725 \\
\hline 2017 & 74 & 16.20 & 186 & 40.72 & 392 & 85.81 & 0.9538 & 0.9189 & 0.8705 \\
\hline
\end{tabular}

Source: authors' processing apud the information from Romania's Statistic Yearbook, 2018

The lowest gap was registered in 2014 , when the salaries obtained by women represented $96.84 \%$ of those obtained by men, in companies with less than 50 employees, given that the ratio of the number of female / male employees was $74.63 \%$. On the average, during the ten years analysed, the ratio of the number of female / male employees is $75.38 \%$ for private enterprises with less than 50 employees, $66.35 \%$ for those with 50-249 employees and $79.26 \%$ for those with over 250 employees, highlighting the more pronounced masculine character of the organizational culture in the enterprises subordinated to the private power. According to Grybaite (2006), "the number of women in the world labour force is growing".

\begin{tabular}{|l|c|c|c|c|c|c|c|c|c|c|}
\hline & & & & & \\
\hline
\end{tabular}




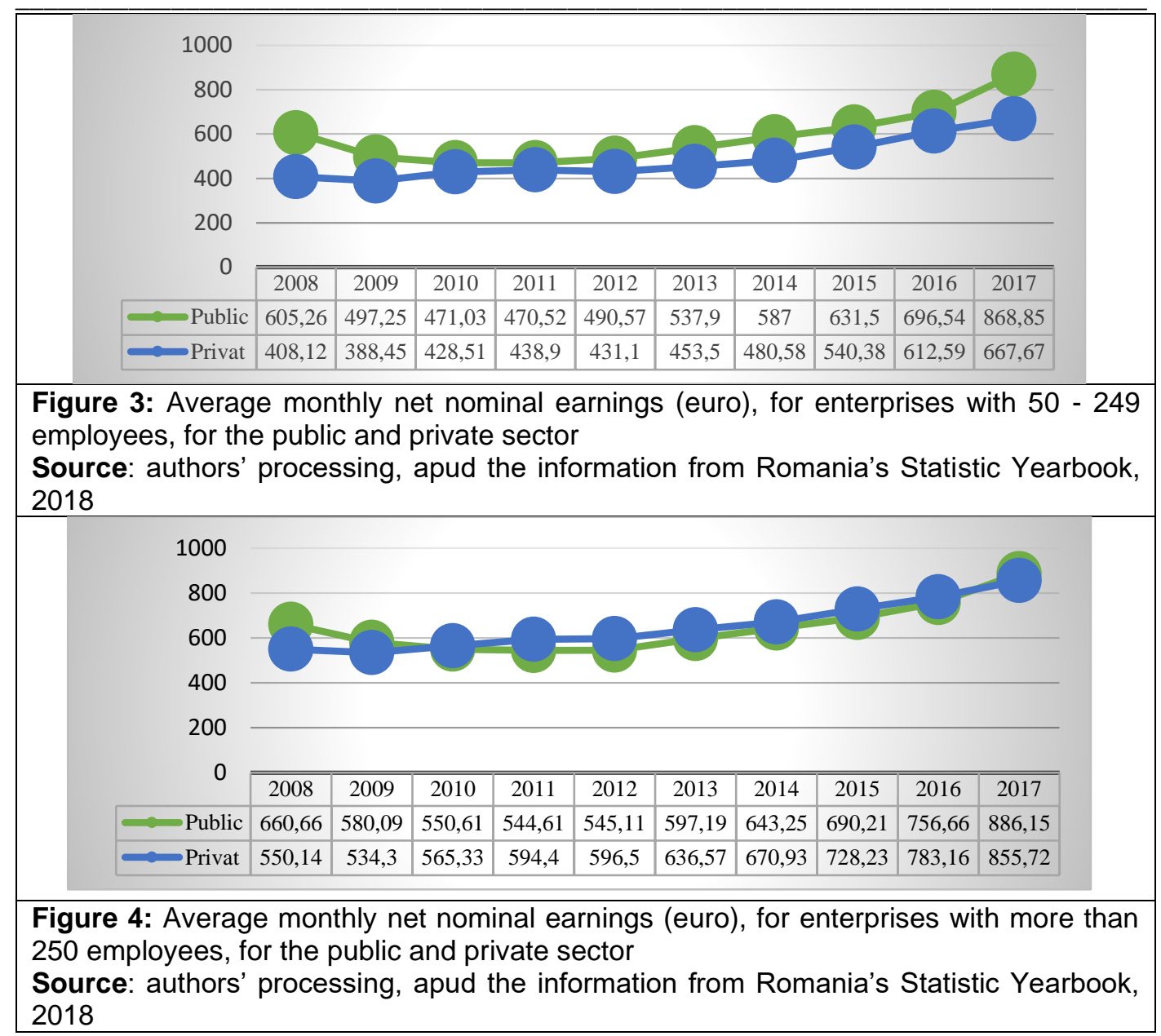

According to the Global Gender Gap Report, "at the dawn of the 2020s, building fairer and more inclusive economies must be the goal of global, national and industry leaders". For small economic agents, which include mainly public administrations and local companies, there is a significant difference between the public and private sectors. This differentiation has been accentuated in the last two years also due to the salary increases that civil servants and medical staff have benefited from.

Regarding the economic agents with more than 250 employees, there is a decrease in the gap between 2008 and 2017, reaching the very situation where, in the autonomous utilities, large public companies, to register higher salary levels than in some multinational companies.

\subsection{The correlation analysis}

The data highlighted in the previous tables were processed and the results obtained are presented below. Thus, for all three criteria C1, C2 and C3, replacing the initial data for two parameters in a regression equation, this becomes:

$$
\text { salary_earnings }=\alpha+\beta \text { * employees_no }+\varepsilon
$$


The parameter $\alpha$ represents the value taken by the resultant variable $Y$ (earnings), when the factorial variable $X$ (number of employees) has the value zero and may have higher or lower relevance depending on the case analysed.

The coefficient $\beta$ represents the regression coefficient, so the value by which the resultant variable $Y$ changes (earnings) when the factorial variable $X$ (number of employees) changes by one unit, its sign determining the level of interdependence between the resultant variable and the factorial variable.

For the analysis of the connection between the two variables, the results of the statistical tests Student and Durbin Watson were used, the table below presenting the values obtained in the program, centralized according to the three basic criteria.

Table 8: The values of the calculated statistical variables

\begin{tabular}{|c|c|c|c|c|c|c|c|}
\hline $\begin{array}{l}\text { C1 - } \\
\text { Company } \\
\text { category }\end{array}$ & $\begin{array}{c}\text { R- } \\
\text { Square } \\
\text { d }\end{array}$ & $\begin{array}{c}\text { F- } \\
\text { Statistic } \\
\mathbf{s}\end{array}$ & $\begin{array}{c}\text { Durbin } \\
- \\
\text { Watson }\end{array}$ & $\begin{array}{c}\mathrm{t}- \\
\text { Statisti } \\
\text { c } \alpha\end{array}$ & $\begin{array}{c}t- \\
\text { Statisti } \\
c \beta\end{array}$ & $\begin{array}{l}\text { Coeffici } \\
\text { ent } \alpha\end{array}$ & $\begin{array}{c}\text { Coeffici } \\
\text { ent } \beta\end{array}$ \\
\hline $\begin{array}{l}\text { Under } 50 \\
\text { employees }\end{array}$ & $\begin{array}{r}0.2672 \\
66\end{array}$ & $\begin{array}{r}2.77102 \\
0^{\star *}\end{array}$ & .263697 & $\begin{array}{r}- \\
0,1770 \\
10\end{array}$ & $\begin{array}{r}1.6646 \\
38\end{array}$ & $\begin{array}{r}- \\
128.764 \\
6\end{array}$ & $\begin{array}{r}0.00087 \\
3\end{array}$ \\
\hline $\begin{array}{l}50-249 \\
\text { employees }\end{array}$ & $\begin{array}{r}0.1302 \\
20\end{array}$ & $\begin{array}{r}1.19772 \\
9^{* *}\end{array}$ & $\begin{array}{r}.425903 \\
* *\end{array}$ & $\begin{array}{r}- \\
0.5209 \\
56\end{array}$ & $\begin{array}{r}1.0944 \\
08\end{array}$ & $\begin{array}{r}2798.76 \\
0\end{array}$ & $\begin{array}{r}0.00256 \\
7\end{array}$ \\
\hline $\begin{array}{l}\text { Over } 250 \\
\text { employees }\end{array}$ & $\begin{array}{r}0.0070 \\
93\end{array}$ & $\begin{array}{r}0.05715 \\
3^{\star *}\end{array}$ & .193384 & $\begin{array}{r}0.9884 \\
44\end{array}$ & $\begin{array}{r}-- \\
0.2390 \\
67\end{array}$ & $\begin{array}{r}2704.12 \\
9\end{array}$ & $\begin{array}{r}0.00030 \\
3\end{array}$ \\
\hline
\end{tabular}

\begin{tabular}{|c|c|c|c|c|c|c|c|}
\hline $\begin{array}{l}\text { C2 - } \\
\text { Activity } \\
\text { sector }\end{array}$ & $\begin{array}{c}\text { R- } \\
\text { Square } \\
\text { d }\end{array}$ & $\begin{array}{c}\text { F- } \\
\text { Statistics }\end{array}$ & $\begin{array}{l}\text { Durbin - } \\
\text { Watson }\end{array}$ & $\begin{array}{c}\mathrm{t}- \\
\text { Statistic } \\
\alpha \\
\end{array}$ & $\begin{array}{c}\mathrm{t}- \\
\text { Statistic } \\
\beta \\
\end{array}$ & $\begin{array}{c}\text { Coefficien } \\
t \alpha\end{array}$ & $\begin{array}{c}\text { Coefficien } \\
t \beta\end{array}$ \\
\hline \multicolumn{8}{|l|}{ Public } \\
\hline $\begin{array}{l}\text { Under } 50 \\
\text { employee } \\
\text { s }\end{array}$ & $\begin{array}{l}0.03780 \\
8\end{array}$ & ${ }_{*}^{0.314349^{*}}$ & $.372607^{*}$ & $\begin{array}{l}0.27656 \\
7\end{array}$ & $\begin{array}{l}0.56066 \\
9\end{array}$ & 596.8565 & 0.010999 \\
\hline $\begin{array}{l}50-249 \\
\text { employee } \\
\text { s }\end{array}$ & $\begin{array}{l}0.01176 \\
7\end{array}$ & $0.095257^{*}$ & $.325604^{*}$ & $\begin{array}{l}1.28924 \\
8\end{array}$ & $\begin{array}{l}- \\
0.30863 \\
8\end{array}$ & 3334.987 & -0.004060 \\
\hline $\begin{array}{l}\text { Over } 250 \\
\text { employee } \\
\text { s }\end{array}$ & $\begin{array}{l}0.32586 \\
1\end{array}$ & $\begin{array}{l}3.866993^{*} \\
\text { * }\end{array}$ & $.461180^{*}$ & $\begin{array}{l}3.48282 \\
0\end{array}$ & $\begin{array}{l}- \\
1.96646 \\
7\end{array}$ & 6391.587 & -0.003594 \\
\hline \multicolumn{8}{|l|}{ Private } \\
\hline $\begin{array}{l}\text { Under } 50 \\
\text { employee } \\
\text { s }\end{array}$ & $\begin{array}{l}0.19905 \\
2\end{array}$ & ${ }_{*}^{1.988163^{*}}$ & $.258940 *$ & $\begin{array}{l}0.13734 \\
3\end{array}$ & $\begin{array}{l}1.41002 \\
2\end{array}$ & 128.1949 & 0.001030 \\
\hline $\begin{array}{l}50-249 \\
\text { employee } \\
\text { s }\end{array}$ & $\begin{array}{l}0.11163 \\
2\end{array}$ & $\begin{array}{l}1.005276^{*} \\
\text { * }\end{array}$ & $.382299^{*}$ & $\begin{array}{l}- \\
0.15605 \\
7\end{array}$ & $\begin{array}{l}1.00263 \\
5\end{array}$ & -389.4214 & 0.002795 \\
\hline $\begin{array}{l}\text { Over } 250 \\
\text { employee } \\
\text { s }\end{array}$ & $\begin{array}{l}0.31058 \\
1\end{array}$ & $\begin{array}{l}3.603981 \text { * } \\
\text { * }\end{array}$ & $\begin{array}{l}.448009^{*} \\
*\end{array}$ & $\begin{array}{l} \\
0.75763 \\
6\end{array}$ & $\begin{array}{l}1.89841 \\
5\end{array}$ & -1886.662 & 0.004080 \\
\hline
\end{tabular}

\begin{tabular}{|c|c|c|c|c|c|c|c|}
\hline $\begin{array}{l}\text { C3 - } \\
\text { Gender of } \\
\text { employee } \\
\text { s }\end{array}$ & $\begin{array}{c}\text { R- } \\
\text { Square } \\
\text { d }\end{array}$ & $\begin{array}{c}\text { F- } \\
\text { Statistics }\end{array}$ & $\begin{array}{c}\text { Durbin } \\
- \\
\text { Watson }\end{array}$ & $\begin{array}{c}t- \\
\text { Statistic } \\
\alpha\end{array}$ & $\begin{array}{c}\mathrm{t}- \\
\text { Statistic } \\
\beta\end{array}$ & $\begin{array}{c}\text { Coefficien } \\
\text { t } \alpha\end{array}$ & $\begin{array}{c}\text { Coefficien } \\
\text { t } \beta\end{array}$ \\
\hline
\end{tabular}


The Annals of the University of Oradea. Economic Sciences

TOM XXX, $1^{\text {st }}$ Issue, July 2021

\begin{tabular}{|c|c|c|c|c|c|c|c|}
\hline \multicolumn{8}{|l|}{ Female } \\
\hline $\begin{array}{l}\text { Under } 50 \\
\text { employees }\end{array}$ & $\begin{array}{c}0.19859 \\
0\end{array}$ & $\underset{*}{1.982412 *}$ & $.20628^{\star}$ & $\begin{array}{l}- \\
0.22499 \\
8\end{array}$ & $\begin{array}{l}1.40798 \\
2\end{array}$ & -269.5678 & 0.002777 \\
\hline $\begin{array}{l}50-249 \\
\text { employees }\end{array}$ & $\begin{array}{c}0.34832 \\
0\end{array}$ & $\underset{*}{4.275959 *}$ & $\underset{*}{.67762^{*}}$ & $\begin{array}{c}- \\
1.56618 \\
2\end{array}$ & $\begin{array}{c}2.06783 \\
9\end{array}$ & -6618.020 & 0.018784 \\
\hline $\begin{array}{l}\text { Over } 250 \\
\text { employees }\end{array}$ & $\begin{array}{c}0.10336 \\
4\end{array}$ & $\underset{*}{0.922234 *}$ & $\underset{*}{.18496^{*}}$ & $\begin{array}{c}- \\
0.26642 \\
9\end{array}$ & $\begin{array}{c}0.96033 \\
0\end{array}$ & -1001.923 & 0.003265 \\
\hline \multicolumn{8}{|l|}{ Male } \\
\hline $\begin{array}{l}\text { Under } 50 \\
\text { employees }\end{array}$ & $\begin{array}{c}0.26565 \\
4\end{array}$ & $\underset{*}{2.894053^{\star}}$ & 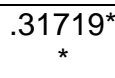 & -.057611 & $\begin{array}{c}1,70119 \\
2\end{array}$ & -53.12792 & 0.002014 \\
\hline $\begin{array}{l}50-249 \\
\text { employees }\end{array}$ & $\begin{array}{c}0.04784 \\
1 \\
\end{array}$ & $\underset{*}{0.401961^{*}}$ & $\begin{array}{c}.30661^{*} \\
*\end{array}$ & $\begin{array}{c}0.03577 \\
6 \\
\end{array}$ & $\begin{array}{c}0.63400 \\
4 \\
\end{array}$ & 119.6808 & 0.003392 \\
\hline $\begin{array}{l}\text { Over } 250 \\
\text { employees }\end{array}$ & $\begin{array}{c}0.19525 \\
5\end{array}$ & ${ }_{*}^{1.941033^{*}}$ & $.30964^{*}$ & $\begin{array}{c}2.39245 \\
0\end{array}$ & $\begin{array}{c}- \\
1.39321 \\
0\end{array}$ & 7254.418 & -0.004009 \\
\hline
\end{tabular}

Source: authors' processing

In cases where $\beta>0$, the link between the factorial variable and the resultant one is a direct one, so when the growth rate of the number of employees has an increasing evolution, the salary gain also increases.

When coefficient $\beta$ is positive, we can encounter the following situations:

$-\beta<1-$ when the influence of the factorial variable on the resulting one is weaker;

- $\beta>1-$ when the influence of the factorial variable on the resulting one is very strong;

$-\beta=1-$ when the resulting variable varies in direct proportion to the variation of the factorial variable.

If the coefficient $\beta<0$, the resulting variable representing earnings is independent of the number of employees.

Based on the data obtained above, we can observe in our case both positive and negative values of the coefficient $\beta$, all positive values being subunitary, which indicates a relative influence on the resultant variable $Y$. In cases where we observe negative values, they correspond, as can be seen from the table above, to large enterprises, and it can be concluded that there is an independent relationship between the two variables in this case. The link observed by calculating the correlation coefficient overall indicates a weak or moderate relationship from this point of view, there are secondary factors that can act on the long-term phenomenon and can show a particular influence on the identified relationship. The statistical verification of the unifactorial model is thus performed based on the Student and Durbin - Watson tests.

In the first case, the tabular value to be used is determined from the table corresponding to the Student distribution, depending on $\mathrm{v}=\mathrm{n}-1$ degrees of freedom and probability $\alpha / 2=$ $0.05 / 2=0.025$. According to the table of values, the value tcritic $=2.262$.

In most cases presented in the results table above, the calculated value of the model parameters is less than or equal to the critical value. (ta, $t \beta<t c r i t i c)$, in which case the null hypothesis is accepted, the $\beta$ estimators not being significantly different from zero. This is the case for private enterprises in particular, as this phenomenon is observed in the case of medium and large enterprises. Most employees in Romania who work in companies with less than 50 employees, are employed with a minimum wage in the economy, noting the phenomenon of "undeclared" pay. There is therefore a high share of employees employed at a minimum, the average approaching the level of the minimum wage in the economy. Its evolution in the time period $2008-2017$ is presented in the graph below: 


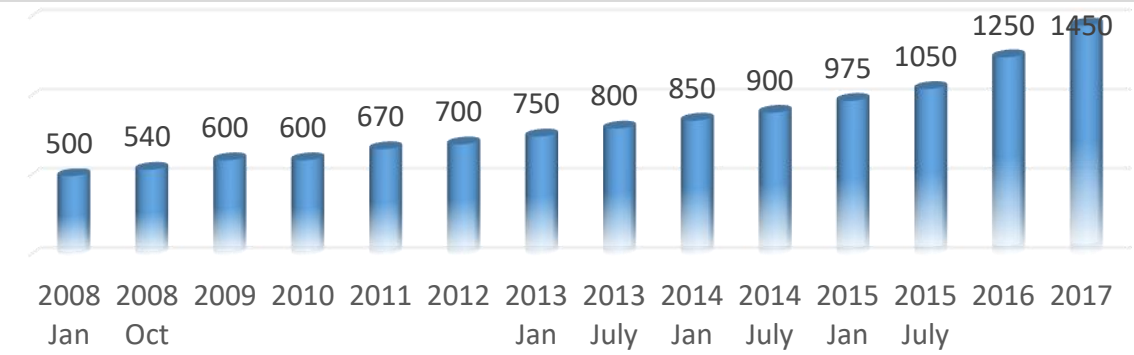

Figure 5: Evolution of the minimum wage in the economy in Romania (lei)

Source: authors 'processing, according to information provided by Manu Consulting

What we can observe according to this information is first of all an increasing evolution, there being years in which the increases took place in two different months. The level corresponding to 2017 is three times higher than that corresponding to the base year, the growth rate being illustrated in the following table:

Table 9: Minimum wage growth rate

\begin{tabular}{|c|c|c|c|c|c|c|c|c|c|c|}
\hline & $\begin{array}{r}2008 / \\
2008 \\
\text { (Oct/Jan) }\end{array}$ & $\begin{array}{r}2009 / \\
2008\end{array}$ & $\begin{array}{r}2010 / \\
2009\end{array}$ & $\begin{array}{r}2011 / \\
2010\end{array}$ & $\begin{array}{r}2012 / \\
2011\end{array}$ & $\begin{array}{r}2013 / \\
2013 \\
\text { (Jul/Jan) } \\
2013 / \\
2012 \\
\text { (Jan) }\end{array}$ & $\begin{array}{r}2014 / \\
2014 \\
\text { (Jul/Jan) } \\
2014 / \\
2013 \\
\text { (Jan } \\
\text { /Jul) }\end{array}$ & $\begin{array}{r}2015 / \\
2015 \\
\text { (Jul/Jan) } \\
2015 / \\
2014 \\
\text { (Jan/Jul) }\end{array}$ & $\begin{array}{r}2016 / \\
2015 \\
\text { (Jul) }\end{array}$ & $\begin{array}{r}2017 / \\
2016\end{array}$ \\
\hline $\begin{array}{l}\text { Growth } \\
\text { rate }\end{array}$ & $\begin{array}{r}13.64 \% \\
8 \%\end{array}$ & $11.11 \%$ & - & $11.6 \%$ & $4.48 \%$ & $\begin{array}{l}7.14 \% \\
6.66 \%\end{array}$ & $\begin{array}{l}6.25 \% \\
5.88 \%\end{array}$ & $\begin{array}{l}8.33 \% \\
7.70 \%\end{array}$ & $19.0 \%$ & $16 \%$ \\
\hline
\end{tabular}

Source: authors' processing

The unifactorial model can, of course, be developed in order to obtain more accurate results, adding other influencing factors (e.g. inflation rate - fig. No. 9) or developing the analysis for a longer period of years, in order to achieve a relevant long-term analysis.

For the Durbin Watson test, two tabular values, one lower and one higher, $\mathrm{dL}$ and $\mathrm{dU}$, are determined from the corresponding statistical tables, depending on the significance level of the test $(0,05)$ and the number of observations $(10), k$ being equal to 1 , being a unifactorial model. In our case, the tabular values are $\mathrm{dL}=0.88$ and $\mathrm{dU}=1.32$.

From the observations introduced and the results obtained, we find that $\mathrm{d}<\mathrm{dL}$ in all situations, regardless of the size class of the company or the type of employees, the hypothesis of autocorrelation of random variables is accepted, the values of the variable being dependent on each other. The model can be corrected, taking into account the influence that the guaranteed minimum wage level on the economy has on the overall evolution, as well as the influence of the legislative framework, the tax system, inflation or internal and external labour migration.

\section{Conclusions}

Approached from the perspective of the concept of sustainability, the Romanian labour market has only partially the characteristic of maintaining a desired trend (increasing employment and living standards of employees), there are variations over time, either due to the effects of the economic crisis (Balcar, Gottvald, 2016) (2008 - 2009), or influences of 
the structure of the internal business environment and the level of training of the workforce, or a manifestation of the effects of electoral cycles on the wage system.

From the perspective of gender differences, the elements of human capital can play an important role in explaining the pay gap. Men and women do not choose the same type of schooling and implicitly the same professions, and from the perspective of experience, women tend to be deficient due to career interruptions caused by raising children.

A common phenomenon on the Romanian labour market is the lack of concordance between the level of education of the employee and the one required by the position in the company's organigramme. Although the legislation provides for higher levels of the minimum wage for graduates, employers, in order to avoid increasing salary costs, do not provide in their organizational structure, positions that require this qualification. This reality does not stimulate young people to pursue higher education and, a situation reflected by the last position occupied by Romania in terms of the number of graduates in the age category 30 $34 \%$, only $26 \%$, in 2017 , well below the European average of $40 \%$.

Following the research aiming at the evolution of the salary differences in the public and private sector, depending on the order of size of the enterprises, by the number of employees and by gender (male / female), the hypotheses subject to validation are:

Salary incomes in public enterprises are higher than in the private sector, the gaps widening over time, a hypothesis confirmed by the analysis of statistical data on net salary incomes in Romania, in the period 2008-2017;

Due to economies of scale and productivity increases, wages in large enterprises (over 250 employees) are higher than in SMEs. Wage differences on the level of large enterprises are insignificant between the public and private sectors. In the analysed period there is a salary gap between the two sectors, in favour of the private one, until 2017, when in the public sector there is an average net monthly salary income of 886 euros, higher than in the private sector, of 855 euros;

Gender discrimination from the perspective of salary income is registered in the case of all types of enterprises. This hypothesis is partially verified, with the exception of the average net salaries obtained in the public sector for medium-sized companies (50-249 employees), from 2013 to 2017 , when the female / male wage ratio is super unitary;

The economic dimension of sustainability is determined by a priority contribution of the private sector to the generation of capital gain, and therefore a primary distribution of income with priority to this sector. This hypothesis is not verified in the case of SMEs, where the average level of the monthly net salary exceeds in the public sector the one registered in the private sector.

The analysis of salary incomes in Romania in the period after its accession to the European Union highlights the incoherence of development strategies from the perspective of the structure of companies in terms of size and public-private relationship, in which the engine of sustainable economic growth should be private investment and therefore a business environment supported by public policies.

\section{References}

1. Balcar, J., Gottvald, J., (2016) "Wage determinants and economic crisis 2008 - 2014: Evidence from the Czech Republic", Ekonomický časopis, 64, c. 1, pp. 3 - 21, Available online at: https://www.sav.sk/journals/uploads/0617094701\%2016\%20Balcar\%20-\%20Gottvald\%20RS-F.pdf 2. Baumol, W.S., Wolf, E.N., (1988) "Productivity growth, convergence and welfare", Econ Rev, 78(5), pp. $1155-1159$, https://www.jstor.org/stable/1807175?seq=1

3. Belas, J., Gavurova, B., Kubalek, J., Kubak, M., (2020) "Discrepancies in perception and evaluation og macroenvironment - sectoral analysis", Journal of Business Economics and Management, 21(4), pp. 1072 - 1092, https://doi.org/10.3846/jbem.2020.12274 
4. Brandolini, A., (2007), "Measurement of income distribution in supranational entities: the case of the European Union", in Jenkins SP, Micklewright J (eds), Inequality and poverty re-examined: Oxford, Oxford University Press, pp.62-83

5. Bhalla, S., (2002), "Imagine there's no country: poverty, inequality and growth in the era of the great recession", JRC Working Papers in Economics and Finance, Washington DC, https://pdfs.semanticscholar.org/a154/3205680897e0736c372b373d02bda320a2e1.pdf

6. Budria, S., Moro - Egido, A., (2014). "Overqualification, skill mismatches and wages in private sector employement in Europe", Technological and economic development of economy, 20(3), pp. 457 - 483, doi: https://doi.org/10.3846/20294913.2014.883341

7. Card, D., Krueger, A.B., (1995), Myth and Measurement: The New Economics of the Minimum Wage, Princeton University Press

8. Chirinko, RS., (1980), "The real wage rate over the business cycle", Rev Econ Stat, 62(3), pp. 459461, https://ideas.repec.org/a/tpr/restat/v62y1980i3p459-61.html

9. Clark, R., (2013) "Convergence in national income distribution", 2013, Soc Forces, 92(2), pp. 413436, doi: $10.1093 /$ sf $/$ sot095

10. Dinga, E.(2009), Economics studies. Contributions of logical, epistemological and methodological analysis (in Romanian in original), Economic Publishing House: Bucharest

11. Dornbusch, R., Fisher, S., (1990), Macroeconomics (in Romanian in original), Sedona Publishing House

12. Gori E., Romolini A., Fissi S., (2018) "Local authorities policies for disseminating gender equality. Evidence from Italy", Transylvanian Review of Administrative Sciences, no. 53 E/2018: 38 - 53, doi: 10.24193/tras.53E.3

13. Gottvald, J., Rievajova, E., Sipikalova, S., (2013), "Determinants of individual wages in the Slovak Republic", Ekonomický časopis, 61 , c. $7, \quad$ pp. 672 - 689 , Available online at https://www.sav.sk/journals/uploads/0621145807\%2013\%20Rievajova\%20a\%20kol.pdf

14. Grybaite, V., (2006), "Analysis of theoretical approaches to gender pay gap", Journal of Business Economics and Management, 7(2), pp. 85 - 91, https://doi.org/10.3846/16111699.2006.9636127

15. Hedija, V., Musil P., (2020), "Wage discrimination against women in Baltic countries", Ekonomický časopis, 68, c. 7, pp. 699 - 713, Available online at https://www.sav.sk/journals/uploads/0722105807\%2020\%20Hedija\%20+\%20SR.pdf

16. Hitka, M., Kozubikova, L., Potkany, M., (2018), "Education and gender - based differences in employee motivation", Journal of Business Economics and Management, 19(1), pp. 80 - 95, https://doi.org/10.3846/16111699.2017.1413009

17. Levy, MJ., (1966) Modernization and the structure of societies: a setting for international affairs, Priceton University Press, https://doi.org/10.1177/106591296702000142

18. Luțac, Gh., (2010), The European labour market: theory and politics in the context of the new economy (in Romanian in original), course of lectures, University Alexandru loan Cuza, Centre of European Studies, 2010

19. Macarie F.C., Moldovan O., (2012) "Gender discrimination in management. Theoretical and empirical perspectives", Transylvanian Review of Administrative Sciences, no. 35 E/2012: 153 - 172 20. Marek L., (2019),"Effect of high wages on average wages in the Czech Republic", Ekonomický časopis, 67, c. 4, pp. $404 \quad-425$, Available online at https://www.sav.sk/journals/uploads/0517112204\%2019\%20Marek\%20+\%20SR.pdf

21. Meyer, JW, Boli, J, Thomas, GM, Ramirez, FO., (1997), "World society and nation state", J Sociol, 103(1), pp. 144-181, doi 10.1086/231174

22. Minică, M., (2004), Microeconomics, Introductory Notions. The market and the system of markets (in Romanian in original), Mirton Publishing House: Timișoara

23. Năstase, C., Chașovschi, C. E., State, M., Scutariu, A. - L., (2019), "Municipal waste management in Romania in the context of the EU. A stakeholders perspective", Technological and economic development of economy, 25 (5), pp. 850 - 876, https://doi.org/10.3846/tede.2019.10295

24. Pauhofova, I., Stehlikova, B., (2018), "Identifying the relationship between unemployment and wage development in the Slovak Republic", Ekonomický časopis, 66, c5, pp. 503 - 521, Available online at https://www.sav.sk/journals/uploads/0530112205\%2018\%20Pauhofova-

Stehlikova\%20+\%20RS.pdf

25. Privarova, M., (2007), "The regulation theory and its relation to main stream of economic thought", Ekonomický časopis, 55, c9, pp. 916 - 928, Available online at: https://www.sav.sk/journals/uploads/0929114009\%2007\%20Privarova.pdf 
26. Radlinska, K., Klonowska - Matynia, M., Jakubowska, A., Kwiatkowski, G. (2020), "Labor hoarding: an old phenomena in modern times? Case study for EU countries", Journal of Business Economics and Management, 21(3), pp. 827 - 889, https://doi.org/10.3846/jbem.2020.12228

27. Rakauskiene, O.G., Chlivickas, E., (2007) "Public finance of Lithuania: gender perspective", Journal of Business Economics and Management, 8(1), pp. 11-27, https://doi.org/10.3846/16111699.2007.9636148

28. Sachs, J, Warner, AM., (1996), "Economic convergence and economic policies,, 1996, NBER Working Paper, 5039, http://www.nber.org/papers/w5039.pdf

29. Samuelson, P., (1958), Economics: An Introductory Analysis, 4th edition, New York: McGraw-Hill 30. Santos, M., Sequeira Tiago, N., (2013), "Skills mismatch and wage inequality: evidence for different countries in Europe", Technological and economic development of economy, 19 (Supplement 1), pp. 5425 - 5453, https://doi.org/10.3846/20294913.2013.880086

31. Sandor S.D., Macarie F.C., Creța S.C., (2011), "Gender equality in the Romanian local public institutions", Transylvanian Review of Administrative Sciences, no. 34 E/2011: 217 - 228

32. Vacas-Soriano, C., Fernández-Marcias, E., Muňos De Bustillo, R., (2019) "Recent trends in wage inequality from an EU perspective: a tale of two convergences", Empirica, Springer, DOI:10.1007/S10663-019-09436-7

33. Torfason, MT, Ingram, P., (2010), "The rise of global democracy: a network account", Social REv 75(3), pp. 355-377, https://www8.gsb.columbia.edu/researcharchive/articles/4476

34. Vargic, B., Luptakova, S., (2003) "Managerial style and its managerial implications for organizations in the Slovak Republic", Journal of Business Economics and Management. IV(1), pp. 36 - 44, doi: 10.3846/16111699.2003.9636036

35. Vaiciukeviciute, A., Stankeviciene, J., Bratcikoviene, N., (2019) "Higher education institutions impact on the economy", Journal of Business Economics and Management, 20(3), pp. 507-525, https://doi.org/10.3846/jbem.2019.10156

36. Zeman J., (2019) "Income distribution and economic growth: empirical results for Slovakia", Ekonomický časopis, 67, c5, pp. $459 \quad-\quad 480$, Available online at https://www.sav.sk/journals/uploads/0712101505\%2019\%20Zeman\%20+\%20SR.pdf

37. Ucak, H., (2012), "The catch-up process of relative wages in European Union New Member States", Ekonomický časopis, 60, c4, pp. $360-370$, Available online at https://www.sav.sk/journals/uploads/0622123104\%2012\%20Ucak-RS.pdf

38. *** "The life of women and men in Europe. A statistical portrait", (2018) Eurostat. Available at https://ec.europa.eu/eurostat/web/products-digital-publications/-/KS-01-18-904

39. *** World Commission on Environment and Development (WCED). Our Common Future, (1987) Oxford University Press: New York, NY, USA. Available at: https://sustainabledevelopment.un.org/content/documents/5987our-common-future.pdf

40. ${ }^{* \star *}$ Agenda for Development; United Nations, (1997) New York, NY, USA

41. ${ }^{* * *}$ Romania's Statistic Yearbook. 2008-2019, National Institute of Statistics, Available at: www.insse.ro

42. ${ }^{* * *}$ World Economic Forum, Global Gender Gap Report, (2020), https://www.weforum.org/reports/gender-gap-2020-report-100-years-pay-equality 